\title{
NCR2 Gene
}

National Cancer Institute

\section{Source}

National Cancer Institute. NCR2 Gene. NCI Thesaurus. Code C104543.

This gene is involved in natural killer cell function. 